\title{
Group representations and the method of sections
}

\author{
BISWADEB DUTTA and N MUKUNDA* \\ Department of Physics, ${ }^{*}$ Centre for Theoretical Studies, Indian Institute of Science, \\ Bangalore 560012 \\ MS received 29 July 1987
}

\begin{abstract}
The unitary representations of the Euclidean and Poincare groups are analysed, using viewpoints suggested by the method of sections, as applied to the monopole problem, and by the method of induced representations,
\end{abstract}

Keywords. Poincart group; induced representations; coset spaces; fibre bundles.

PACS Nos 0220; 0240; $11 \cdot 30$

\section{Introduction}

The quantum mechanical motion of a charged particle in the field of a static magnetic monopole was first discussed by Dirac (Dirac 1931). The vector potential of such a magnetic field has an unavoidable string singularity at least semi-infinite in extent. Dirac showed that as a kinematic requirement every wave function for the charged particle must necessarily vanish along the string. Consistency of this requirement and of behaviour near the string with quantum mechanical principles led Dirac to the quantization condition

$$
e g=\frac{\pi}{n} \hbar
$$

In an effort to eliminate the string, Wu and Yang (Wu and Yang 1975) developed another way of describing quantum mechanical states in the above situation. The three-dimensional space around and excluding the monopole, $S^{2} \times \mathbb{R}+$, is expressed as the union of two open, overlapping, topologically trivial regions $R_{a}$ and $R_{b}$. For instance, $R$, omits the negative z-axis while $R_{b}$ omits the positive part. In each of the regions $R_{a}$ and $R_{b}$ a corresponding singularity free vector potential $A, A_{b}$ can be chosen; in the overlap $R_{a} \cap R_{b}$ where both are defined, they are related by a gauge transformation. Each quantum mechanical state is then described by a pair of singularity-free wave functions $\psi_{a}, \psi_{b}$ defined on $R_{a}, R_{b}$ respectively. Over $R_{a} \cap R_{b}, \psi_{a}$ and $\psi_{b}$ are related by the same gauge transformation that relates $\boldsymbol{A}_{a}$ to $\boldsymbol{A}_{b}$. This transition rule connecting $\psi_{a}$ and $\psi_{b}$ in the overlap is kinematic in nature, being the same for all states (in the given monopole field). The requirement that it be well-defined, i.e. singlevalued, leads us back to the quantization condition (1).This so-called method

\footnotetext{
* Jawaharlal Nehru Fellow.
} 
Biedenharn and Louck 1981). The non-Abelian generalization of the magnetic monopole field is discussed by Coleman, and by Goddard and Olive (Coleman 1981; Goddard arid Olive 1978).

In another independent approach to the string problem, it has been shown (Balachandran et al 1980) that the string can be avoided altogether if one works with a degenerate Lagrangian based on an augmented configuration space $S U(2) \times \mathbb{R}_{+}$rather than the physically accessible $S^{2} \times \mathbb{R}+$. Here $S U(2)$ is viewed as a principal fibre bundle, with structure group $\mathrm{U}(1)$, on the base $S^{2}$, the sphere of all possible directions emanating from the monopole in physical space. $S^{2}$ is itself the space of (right) cosets

$$
|\Psi\rangle \rightarrow \psi(g) \in \mathscr{V} \text { for all } g \in G,
$$

obeying a covariance condition with respect to $H$ :

$$
\psi(g h)=D\left(h^{-1}\right) \psi(g) \text { for all } h \in H .
$$

On such $|\Psi\rangle$, we define a representation $U(\cdot)$ of $\mathrm{G}$ by the action

$$
U\left(g_{1}\right)|\Psi\rangle=\left|\Psi^{\prime}\right\rangle: \quad \psi^{\prime}(g)=\psi\left(g_{1}^{-1} g\right) .
$$

It is immediate that this action preserves the covariance condition (3) and possesses the representation property. Moreover, the covariance condition ensures that $(\psi(g), \psi(g))_{\mathcal{Y}}$ is constant over each (right)coset $g H$ in G. At this stage we introduce the symbol $\Sigma$ to generically denote a (right) coset space, and $p$ for a general point in $\Sigma$ :

$$
\Sigma=G / H, \quad p \in \Sigma \text {. }
$$

The distinguished point in $\Sigma$ corresponding to the coset $H$ containing the identity element will be denoted by $p^{(0)}$. One can then make the representation $U(\cdot)$ of $\mathrm{G}$ given by (4) a unitary one by defining a suitable invariant inner product

$$
\langle\Psi \mid \Psi\rangle=\int_{\Sigma} \mathrm{d} \mu(p)(\psi(g), \psi(g))_{\mathscr{\gamma}},
$$


and thus also completing the definition of the Hilbert space $\mathscr{H}$. This unitary representation of $\mathrm{G}$ is said to be induced from the UIR $\mathrm{D}(\cdot)$ of $H$ originally given on $\mathscr{V}$.

The UIR's of the Euclidean group E(3)(Pauli 1965), i.e. the group of rigid motions in three-dimensional space and of the inhomogeneous Lorentz group, or Poincare group $\mathscr{P}$ (Wigner 1939; Wightman 1960) of special relativity, are of basic significance in quantum mechanics. Even though these representations have been known and are familiar since a long time, the method of induced representations seems the most elegant and economical way of arriving at them.

It is clear that each $\psi(g)$ obeying the covariance condition (3), though not constant over a coset, is determined all over a coset if its "value" at one representative point in the coset is known. Now $G$ acts on $\Sigma=\boldsymbol{G} / \boldsymbol{H}$ transitively by left multiplication:

$$
g \in G, p \in \Sigma \rightarrow p^{\prime}=g p \in \Sigma .
$$

Elements of $H$ are distinguished by the property that they leave $p^{(0)}$ invariant:

$$
h p^{(0)}=p^{(0)} \text { for all } h \in H .
$$

To choose a coset representative for each coset in $\mathrm{G}$ is to pick an element $l(p) \in \mathrm{G}$ for each $p \in \Sigma$ such that

$$
l(p) p^{(0)}=P
$$

in the sense of the action (7) of G on $\Sigma$. The "independent information" contained in $\psi(g)$ is then the collection of its values at the elements $l(p) \in \mathrm{G}$ :

$$
\psi(l(p)) \equiv \varphi(p) \in \mathscr{V}
$$

In this sense, each $\psi(g)$ is essentially a function $\varphi(p)$ on $\Sigma$ with values in $\mathscr{V}$, and the action of $U(g)$ on $|\Psi\rangle$ can be expressed directly in terms of $\varphi(p)$ :

$$
\begin{aligned}
& U(g)|\Psi\rangle=\left|\Psi^{\prime}\right\rangle \Rightarrow \\
& \varphi^{\prime}(p)=D\left(l(p)^{-1} g l\left(g^{-1} p\right)\right) \varphi\left(g^{-1} p\right)
\end{aligned}
$$

If an orthonormal basis for $\mathscr{V}$ is indexed by letters $a, \beta, \ldots$, the components $\varphi_{\alpha}(p)$ of $\varphi(p) \in \mathscr{V}$ can be written as

$$
\varphi_{\alpha}(p)=\langle p, \alpha \mid \varphi\rangle
$$

so that $\mid p, a)$ is an (ideal) orthogonal basis for $\mathscr{H}$. Then (11) can be reexpressed as

$$
\begin{gathered}
U(g)|p, \alpha\rangle=\sum_{\beta} D_{\beta \alpha}(h(g, p))|g p, \beta\rangle, \\
h(g, p)=l(g p)-{ }^{1} g l(p) \in H .
\end{gathered}
$$

The inner products among the (ideal)basis vectors $\mid p, a$ ) are fixed by (6). This form of an induced group representation is familiar from the Wigner construction of the UIR's of $\mathscr{P}$, in which context the element $h(g, p)$ in $H$ in (13) is called the "Wigner rotation".

Given the above general structure, the following questions naturally arise: When can the coset representatives $l(p)$ be chosen in a smooth and singularity-free way $a 1$ over $\Sigma$ ? 
Since the Wigner rotation is a function of both $g$ and $p$ in general, what is the maximum simplification that can be achieved in its form, at least when $g$ is an element $h \in H$ ? In this paper we address ourselves to these questions in the context of the representations of $\mathbf{E}(\mathbf{3})$ and $\mathscr{P}$.

The attempt to choose $l(p)$ for each $p \in \Sigma$ obeying (9)is evidently an attempt to find a section when $\mathrm{G}$ is viewed as a principal fibre bundle on $\Sigma=G / H$ as base and with $H$ as fibre.It follows that a globally smooth choice of $l(p)$ is possible when and only when this bundle is trivial. If it is nontrivial, any attempt to find an $l(p)$ for all $p$ is bound to encounter singularities,just like the vector potential in the monopole problem. As with the $\mathrm{Wu}$-Yang treatment of quantum mechanical states in the presence of a monopole, we can avoid such "kinematic singularities" in the induced representations of G by expressing the base $\Sigma$ as the union of (at least) two open topologically trivial regions $R_{a}$, $R_{b}$ :

$$
\Sigma=R_{a} \cup R_{b}
$$

and choosing $l_{a}(p), l_{b}(p)$ over $R_{a}, R_{b}$ in a singularity-free way. This means that over each of $R_{a}$ and $R_{b}$, the bundle structure can be trivialised; and the portions of $\mathrm{G}$ sitting on top of $R_{a}$ and $R_{b}, G_{a}$ and $G_{b}$ say, are homeomorphic to the products $R_{a} \times H, R_{b} \times H$ respectively. For $p$ in the overlap we necessarily have:

$$
\begin{aligned}
& p \in R_{a} \cap R_{b}: l_{b}(p)=l_{a}(p) h_{T}(p), \\
& h_{T}(p) \in H .
\end{aligned}
$$

Then $|\Psi\rangle \in \mathscr{H}$ is specified by a pair of functions $\varphi_{a}(p), \varphi_{b}(p)$ defined on $R_{a}, R_{b}$ respectively and taking values in $\mathscr{V}$ :

$$
\begin{array}{ll}
\varphi_{a}(p) \equiv \psi\left(l_{a}(p)\right), & p \in R_{a} \\
\varphi_{b}(p) \equiv \psi\left(l_{b}(p)\right), & p \in R_{b} .
\end{array}
$$

In the overlap we have the kinematic transition rule

$$
p \in R_{a} \cap R_{b}: \varphi_{b}(p)=D\left(h _ { T } ( p ) ^ { - 1 } \left(\varphi_{a}(p) .\right.\right.
$$

In principle it is straightforward to derive equations which give the effect of $U(g)$ on a pair $\left(\varphi_{a}(p), \varphi_{b}(p)\right)$ to give a new pair $\left(\varphi_{a}^{\prime}(p), \varphi_{b}^{\prime}(p)\right)$ also obeying (17). The important point is that in order to avoid string-like singularities in $l(p)$ and the accompanying Wigner rotations, one must pay the price of working with sections rather than globally defined wave functions, at least if the intention is to operate directly with the independent information in $\psi(g)$.

When $\mathrm{G}$ is a nontrivial bundle over $G / H$, one can pose the following further questions: (a) Can the two (or more) open sets $R, R_{b}$ in $\Sigma$ be chosen so that each is carried into itself under action by elements of $\mathrm{H}$ ? (b) If the answer is in the affirmative, can the choices of $l_{a}(p), l_{b}(p)$ be made so that at least for elements $h \in H$ the p-dependence in the Wigner rotation is eliminated

$$
\begin{array}{ll}
l_{a}(h p)=h l_{a}(p) h^{-1}, & p \in R_{a} \\
l_{b}(h p)=h l_{b}(p) h^{-1}, & p \in R_{b} ?
\end{array}
$$


The motivation is that if this can be achieved, then for elements $h \in H$ the action of $U(h)$ on $|\varphi\rangle$ would be simple and would use just the UIR $D(\cdot)$ of $H$ originally chosen:

$$
\begin{aligned}
& U(h)|\varphi\rangle=\left|\varphi^{\prime}\right\rangle \\
& \varphi_{a}^{\prime}(p)=D(h) \varphi_{a}\left(h^{-1} p\right), \\
& \varphi_{b}^{\prime}(p)=D(h) \varphi_{b}\left(h^{-1} p\right) .
\end{aligned}
$$

This form, when it can be achieved, is called the Shirokov-Foldy form (see Mukunda 1970).

We shall study the UIR's of $E(3)$ and $\mathscr{P}$ from the point of view of answering the questions raised above. Since our intention is not to derive these well known representations by the method of induction but rather to examine them to answer the questions posed above, we shall be brief in the identifications of $\mathrm{G}, H$ and $\Sigma$ in each case, and shall go on directly to study the relevant topological aspects. Furthermore, we shall be more interested in studying the way the operators $U(h)$ act on $\varphi_{a}(p), \varphi_{b}(p)$ for $h \in H$, and not in the manner in which $U(g)$ for general $g \in G$ mix these $\varphi$ 's.

The paper is organized as follows, In $\S 2$ we study the UIR's of $E(3)$, or rather of its two-fold covering group. The results are very similar to what are known for the treatments of the monopole problem. Section 3, devoted to the UIR's of the Poincaré group, begins with notational preliminaries and general considerations common to all the three types of UIR's; we then study in detail the timelike, spacelike, and lightlike UIR's in that sequence, since that is the way the complexities increase. It will be seen that from the topological point of view, the lightlike case is the most intricate. Section 4 is devoted to a summary of our results and some remarks.

\section{Representations of the Euclidean group}

In this section $\mathrm{G}$ will be the (two-fold)covering group of the Euclidean group $E$ (3). It is the semidirect product of $S U(2)$ by the group $T(3)$ of translations in three-dimensional space:

$$
G=\mathrm{SU}(2) \times T(3)
$$

A general element in $\mathrm{G}$ is

$$
\begin{aligned}
& g=(u, \mathbf{a}), \\
& u \in \operatorname{SU}(2), \quad \mathbf{a} \in \mathbb{R}^{3},
\end{aligned}
$$

and the composition law is

$$
\left(u^{\prime}, \mathbf{a}^{\prime}\right)(u, \mathbf{a})=\left(u^{\prime} u, \mathbf{a}^{\prime}+R\left(u^{\prime}\right) \mathbf{a}\right) .
$$

The rotation $R(u) \in \mathrm{SO}(3)$ determined by $u \in \mathrm{SU}(2)$ is defined as usual by

$$
u \sigma \cdot x u{ }^{1}=\sigma \cdot R(u) \mathrm{x},
$$

where $\boldsymbol{\sigma}$ is the set of Pauli matrices and $\mathbf{x}$ is a real three-vector. 
The subgroup $H \subset \mathrm{G}$ is the semidirect product of $U(1)$ by $T(3)$, where $U(1)$ is the diagonal sub-group in $\mathrm{SU}(2)$ :

$$
\begin{aligned}
& U(1)=\left\{h(\theta)=\exp \left(\frac{i}{2} \theta \sigma_{3}\right) / 0 \leqslant \theta<4 \pi\right\} \subset S U(2), \\
& H=U(1) \times T(3)=\left\{(h, \mathbf{a}) / h \in U(1), \mathbf{a} \in \mathbb{R}^{3}\right\} .
\end{aligned}
$$

(Note that we have departed from the notation in the introduction and are using $h$ for an element in the homogeneous part $H$, of $H$, rather than in $H$; this is convenient and adequate in dealing with $\mathrm{E}(3)$ as well as with $\mathscr{P})$. Therefore the coset space $\Sigma=G / H$ is

$$
\Sigma=\mathrm{SU}(2) \times \mathrm{T}(3) / \mathrm{U}(1) \times \mathrm{T}(3) \simeq \mathrm{SU}(2) / \mathrm{U}(1)=S^{2} .
$$

Thus the general point $p \in \Sigma$ can be taken to be a real three-vector $\mathbf{p}$ of fixed (say unit) length. The distinguished point $\mathbf{p}^{(0)}$ is the "north pole" of $S^{2}$ :

$$
\mathbf{p}^{(0)}=(0,0,1) \text {. }
$$

This corresponds to setting up the canonical map $\pi: G \rightarrow \Sigma$ by the rule

$$
\pi:(u, \mathbf{a}) \in G \rightarrow u \sigma_{3} u^{-1}=\boldsymbol{\sigma} \cdot \mathbf{p}, \quad \mathbf{p} \in S^{2} .
$$

The (left)action of $g=(u, \mathbf{a}) \in G$ on $\Sigma$ amounts to rotating $\mathbf{p}$ by the homogeneous part $u$ of $g$ :

$$
\mathbf{p}^{\prime}=g \mathbf{p}=R(u) \mathbf{p}, \text { independent of } \mathbf{a} .
$$

This can be seen by writing out the subset of elements of $\mathrm{G}$ making up the coset $g_{0} H$ for some $g_{0} \in G$, and seeing how this subset changes upon left multiplication by $g$.

For the inducing procedure one takes the space $\mathscr{V}$ to be one-dimensional, and the UIR $D(\cdot)$ of $H$ to be

$$
\begin{aligned}
& (h(\theta), \mathbf{a}) \rightarrow \exp \left(\operatorname{im} \theta+\mathbf{i a} \cdot \mathbf{p}^{(0)}\right), \\
& m=0, \pm 1 / 2, \pm 1, \ldots
\end{aligned}
$$

The representation $U(\cdot)$ of $\mathrm{G}$ that results is irreducible. It is characterized by helicity $m$, and magnitude of momentum equal to unity. Here the momentum operators are identified, as usual, as the hermitian generators $\mathbf{p}$ of the translations $T(3) \subset \mathrm{G}$.

For all practical purposes, in dealing with the topological aspects and considering choices of $R_{a, b}, l_{a, b}(p)$, etc., one can restrict oneself to the homogeneous parts $G_{0}, H$, i.e. $\mathrm{SU}(2), \mathrm{U}(1)$ of $\mathrm{G}$ and $H$. Thus $l_{a, b}(p)$ can be chosen to lie in $\mathrm{SU}(2)$. Since $\mathrm{SU}(2)$ is a nontrivial U(1)bundle over $S^{2}$, we do need (at least) two open subsets $R, R_{b}$ of $\Sigma=S^{2}$ to avoid singularities in $l(p)$. Now the action of $H_{0}$ on $\Sigma$ is seen from (28) to consist of rotations about the $\mathrm{z}$-axis. As in the $\mathrm{Wu}$-Yang treatment of the monopole problem, we therefore choose

$$
\begin{aligned}
& R_{a}=S^{2}-\{(0,0,-1)\}, \\
& R_{b}=S^{2}-\{(0,0,1)\} .
\end{aligned}
$$


This secures invariance of each of $R, R$, under $H_{0}$ action. Thereforeit is meaningful to ask for $l_{a}(\mathbf{p}) \in \mathrm{SU}(2)$ for all $\mathbf{p} \in R_{a}$ such that

$$
\begin{aligned}
& l_{a}(\mathbf{p}) \mathbf{p}^{(0)}=\mathbf{p} \\
& l_{a}(h(\theta) \mathbf{p})=h(\theta) l_{a}(\mathbf{p}) h(\theta)^{-1}, \quad 0 \leqslant \theta<471
\end{aligned}
$$

As is known, such $l_{a}(\mathbf{p})$ can be found, for example

$$
\begin{aligned}
l_{a}(\mathbf{p}) & =\left[2\left(1+p_{3}\right)\right]^{-1 / 2}\left(1+\mathbf{p} \cdot \boldsymbol{\sigma} \sigma_{3}\right), \\
\mathbf{p} & \in R_{a} .
\end{aligned}
$$

We next ask if an $l_{b}(\mathbf{p}) \in \mathrm{SU}(2)$ can be found obeying the same (31(a),(b)) but now for $\mathbf{p} \in R_{b}$. The answer is that this is impossible, and for the following reason. Consider the point $(0,0,-1) \in R_{b}$, which has the same (homogeneous)stability group $U(1) \subset \mathrm{SU}(2)$ as $\mathbf{p}^{(0)}$. If a singularity-free choice of $l_{b}(\mathbf{p}) \in \mathrm{SU}(2)$ over $R_{b}$ were available obeying $(31 \mathrm{~b})$, then at $\mathbf{p}=(0,0,-1)$ we would have

$$
l_{b}((0,0,-1))=h(\theta) l_{b}((0,0,-1)) h(\theta)^{-1}, \quad 0 \leqslant \theta<4 \pi .
$$

This forces $l_{b}((0,0,-1))$ to belong to the commutant of $U(1)$ in $\operatorname{SU}(2)$, which is $U(1)$ itself. But then $l_{b}((0,0,-1))$ cannot satisfy (31a):

$$
\begin{aligned}
& l_{b}((0,0,-1)) \in U(1) \Rightarrow \\
& I_{1}((0,0,-1)) \mathbf{p}^{(0)}=\mathbf{p}^{(0)} \neq \quad(0,0,-1) .
\end{aligned}
$$

Thus the twin conditions (31) can be obeyed on $R_{a}$ but not on $R_{b}$, and it is not possible to bring the action of $U(1)$ to the simple form indicated in (19).

Under these circumstances, a possible natural choice for $l_{b}(\mathbf{p})$ is

$$
l_{b}(\mathbf{p})=l_{a}(-\mathbf{p}) \cdot i \sigma_{2}, \quad \mathbf{p} \in R_{b} .
$$

This is based on the fact that

$$
\mathrm{p} \in R_{b} \Leftrightarrow-\mathrm{p} \in R_{a}
$$

and that the rotation $R\left(i \sigma_{2}\right)$ carries $\mathbf{p}^{(0)}$ to $-\mathbf{p}^{(0)}$. With this choice for $l_{b}(\mathbf{p})$ we find that the "transition element" $h_{T}(\mathbf{p}) \in U(1)$ determined by (15) in $R_{a} \cap R_{b}$ is

$$
\begin{aligned}
& \begin{aligned}
h_{T}(\mathbf{p}) & =l_{a}(\mathbf{p})-{ }^{1} l_{b}(\mathbf{p}) \\
& =\exp \left(-i \phi \sigma_{3}\right) \\
& =h(-2 \phi),
\end{aligned} \\
& \phi=\text { azimuth of } \mathbf{p} \in R_{a} \cap R_{b} .
\end{aligned}
$$

And instead of (31b) obeyed by $l_{a}(\mathbf{p}), l_{b}(\mathbf{p})$ obeys

$$
l_{b}(h(\theta) \mathbf{p})=h(\theta) l_{b}(\mathbf{p}) h(\theta), \quad 0 \leqslant \theta<4 \pi .
$$

Therefore if the helicity $m$ UIR of $\mathrm{G}$ is set up in the language of sections, the functions 
$\varphi_{a}(\mathbf{p}), \varphi_{b}(\mathbf{p})$ defined over $R_{a}, R_{b}$ respectively will be related by the transition rule

$$
\begin{aligned}
& \varphi_{b}(\mathbf{p})=\exp (2 \operatorname{im} \phi) \varphi_{a}(\mathbf{p}), \\
& \phi=\text { azimuth of } \mathbf{p} \in R_{a} \cap R_{b} .
\end{aligned}
$$

And the effect of $U(h(\theta))$ on these sections is

$$
\begin{aligned}
& U(h(\theta))\left|\varphi^{\prime}\right\rangle=\left|\varphi^{\prime}\right\rangle \\
& \varphi_{a}^{\prime}(\mathbf{p})=\exp (i m \theta) \varphi_{a}\left(R\left(\exp \left(\frac{-i}{2} \theta \sigma_{3}\right)\right) \mathbf{p}\right) \\
& \varphi_{b}^{\prime}(\mathbf{p})=\exp (-i m \theta) \varphi_{b}\left(R\left(\exp \left(\frac{-i}{2} \theta \sigma_{3}\right)\right) \mathbf{p}\right)
\end{aligned}
$$

The essential point we have brought out is that it is impossible to make $\varphi_{a}(\mathbf{p})$ and $\varphi_{b}(\mathbf{p})$ transform identically under the distinguished subgroup $U(1) \subset \mathrm{SU}(2)$, provided $m \neq 0$.

We conclude with the remark that in this problem the analogue of the Dirac quantization condition (1) is that, in order that the transition rule (39) be well defined and single-valued over $R_{a} \cap R_{b}, m$ is restricted to the values $0, \pm 1 / 2, \pm 1, \ldots$

\section{Representations of the Poincaré group}

\subsection{General considerations}

The UIR's of the covering group $\bar{P}$ of the Poincare group $\mathscr{P}$ have a greater variety than in the case of E(3). As shown by Wigner (Wigner 1939; Wightman 1960), they are of three distinct types: timelike $(t)$,spacelike(s), and lightlike $(l)$, depending on the nature of the eigenvalues of the energy momentum operators. (We ignore those UIR's of $\mathscr{P}$ where the translations are trivially realized). We write $p^{\mu}$ for these eigenvalues, and for convenience we normalize $p^{\mu} p_{\mu}$ to -1 in the $t$ and to +1 in the $s$ cases. (We use the metric $g_{00}=-1$ ). In the $t$ and $l$ cases, $p^{0}$ may be taken to be positive definite.

The group $\overline{\mathscr{P}}$ is the semidirect product of $\operatorname{SL}(2, \mathrm{C})$ with the space-time translation group T(4): (our notations for $\mathscr{P}$ are standard; see, for example, Mukunda 1970):

$$
\overline{\mathscr{P}}=\mathrm{SL}(2, \mathrm{C}) \times T(\mathbf{4})
$$

Elements of $\overline{\mathscr{P}}$ and their composition law are:

$$
\begin{aligned}
& g=\left(A, a^{\mu}\right) \in \overline{\mathscr{P}}, \\
& A \in \mathrm{SL}(2, C), \quad a^{\mu} \in \mathbb{R}^{4} ; \\
& \left(A^{\prime}, a^{\prime \mu}\right)\left(A, a^{\mu}\right)=\left(A^{\prime} A, a^{\prime \mu}+\Lambda\left(A^{\prime}\right)^{\mu}{ }_{\nu} a^{\nu}\right) .
\end{aligned}
$$

The homogeneous proper Lorentz transformation $\Lambda(A) \in \operatorname{SO}(3,1)$ determined by $A$ is fixed by

$$
\begin{aligned}
& A \sigma_{\mu} x^{\mu} A^{+}=\sigma_{\mu} \Lambda(A)^{\mu}{ }_{v} x^{\nu} \\
& \sigma \cdot x \equiv \sigma_{\mu} x^{\mu}=x^{0} \cdot \mathbf{1}+\mathbf{x} \cdot \boldsymbol{\sigma}
\end{aligned}
$$


Each type of UIR of $\overrightarrow{\mathscr{P}}$ is associated with a particular subgroup of $\operatorname{SL}(2, C)$ :

$$
\begin{aligned}
& t: \operatorname{SU}(2)=\left\{\left(\begin{array}{cc}
\lambda & \mu \\
-\mu^{*} & \lambda^{*}
\end{array}\right) /|\lambda|^{2}+|\mu|^{2}=1\right\} ; \\
& s: \operatorname{SU}(1,1)=\left\{\left(\begin{array}{cc}
\lambda & \mu \\
\mu^{*} & \lambda^{*}
\end{array}\right) /|\lambda|^{2}-|\mu|^{2}=1\right\} ; \\
& l: E(2)=\left\{\left(\begin{array}{cc}
\lambda & \mu \\
0 & \lambda^{*}
\end{array}\right) /|\lambda|=1\right\} .
\end{aligned}
$$

For the inducing construction, the necessary subgroups of $\ddot{\mathscr{P}}$ are the semidirect products of these subgroups of SL $(2, \mathrm{C})$ with $T(4)$ :

$$
H_{t \text { or } s \text { or } l}=(\mathrm{SU}(2) \text { or } \mathrm{SU}(1,1) \text { or } E(2)) \times T(4)
$$

The coset spaces $\Sigma_{t, s, l}=\overline{\mathscr{P}} / H_{t, s, l}$ can be realized in Minkowski space as

$$
\begin{aligned}
& \Sigma_{t}=\left\{p^{\mu} / p^{\mu} p_{\mu}=-1, p^{\circ}>0\right\} \\
& \Sigma_{s}=\left\{p^{\mu} / p^{\mu} p_{\mu}=1\right\}, \\
& \Sigma_{l}=\left\{p^{\mu} / p^{\mu} p_{\mu}=0, \quad p^{0}>0\right\} .
\end{aligned}
$$

$\overrightarrow{\mathscr{P}}$ is a principal fibre bundle over $\Sigma_{t, s, l}$ with structure group $H_{t, s, l}$. The canonical projection map in each case can be given in a manner similar to (27), and once again only the homogeneous part $\boldsymbol{A}$ in the general element $\left(A ; a^{\mu}\right)$ is involved:

$$
\begin{aligned}
& \pi:\left(A, a^{\mu}\right) \in \overline{\mathscr{P}} \rightarrow A\left(\mathbb{1} \text { or } \sigma_{3} \text { or } 1+\sigma_{3}\right) A^{+}=\sigma_{\mu} p^{\mu}, \\
& p^{\mu} \in \Sigma_{t \text { or } s \text { or } l} ; \\
& p^{(0) \mu}=(1,0,0,0) \text { or }(0,0,0,1) \text { or }(1,0,0, \mathrm{I}) .
\end{aligned}
$$

The distinguished points $p^{(0)}$ in the three cases are also listed. The action of $g=\left(A, a^{\mu}\right) \in \overline{\mathscr{P}}$ on a point $p^{\mu} \in \Sigma_{t, s, t}$ involves only $\boldsymbol{A}$ :

$$
g\left(A, a^{\mu}\right): p^{\mu} \rightarrow p^{\prime \mu}=\Lambda(A)^{\mu}{ }_{\nu} p^{\nu}
$$

Therefore the stability group of $p^{(0)}$ in each case is the corresponding $H$ given in (45).

To see when we have a nontrivial bundle structure, it suffices to look at the homogeneous parts. The topological structures of $\mathrm{SL}(2, C)$, its relevant subgroups and the $\Sigma$ 's are:

$$
\begin{aligned}
& \mathrm{SL}(2, C) \simeq S^{3} \times \mathbb{R}^{3} ; \\
& \mathrm{SU}(2) \simeq S^{3}, \quad \Sigma_{t} \simeq \mathbb{R}^{3} ; \\
& \mathrm{SU}(1,1) \simeq S^{1} \times \mathbb{R}^{2}, \quad \Sigma_{s} \simeq S^{2} \times \mathbb{R} ; \\
& \mathrm{E}(2) \simeq S^{1} \times \mathbb{R}^{2}, \quad \Sigma_{l} \simeq S^{2} \times \mathbb{R} .
\end{aligned}
$$


It is immediately clear that $\not \partial$ is a trivial $H$, bundle over $\Sigma_{t}$, but a nontrivial $H_{s \text { or }}$ bundle over $\Sigma_{s \text { or } l}$. This is the same as the statement that $\mathrm{SL}(2, \mathrm{C})$ is a trivial SU(2) bundle over $\Sigma_{t}$, and a nontrivial $S U(1,1)(E(2))$ bundle over $\Sigma_{s}\left(\Sigma_{l}\right)$. Therefore in the $s$ and $l$ cases we will have to express $\Sigma$ as the union of (at least) two open subsets $R_{a}$ and $R_{b}$, as in the $\mathrm{E}(3)$ case, in order to have singularity-freeexpressions for the coset representatives $l(p)$; but $\Sigma_{t}$ need not be split up in this way. Even in the $t$ case, however, we can ask if $l(p)$ can be chosen so as to obey (18) with respect to $\mathrm{SU}(2)$.

As in the previous section, we use $h, h, \ldots$, to denote elements in the homogeneous parts $\mathrm{SU}(2), \ldots$, of $H, \ldots$, rather than in $H \ldots$. To obtain the various UIR's of $\overrightarrow{\mathscr{P}}$ via the inducing construction, we pick a UIR $D(\cdot)$ of $\mathrm{SU}(2), \ldots$ in a Hilbert space $\mathscr{r}$ and extend it to a UIR of $H$. . by:

$$
\left(h, a^{\mu}\right) \in H \rightarrow D(h) \exp \left(i a^{\mu} p_{\mu}^{(0)}\right) .
$$

Here the standard momenta $p^{(0)}$ are as given in (47). For the $t$ case, $\mathscr{W}$ is of finite dimension; for the s case, $\mathscr{V}$ is of infinite dimension for a nontrivial $D(\cdot)$; and for the $I$ case, $\mathscr{V}$ is of dimension one or infinity according as the "translations" in $\mathrm{E}(2)$ are realized trivially or nontrivially.

With this general background we can examine now the three types of UIR's of $\overline{\mathscr{P}}$ from the point of view of the questions posed in $\S 1$. The characteristic variations in the answers as we look at the cases $t, \mathrm{~s}$ and $l$ in sequence will be brought out.

\subsection{The timelike representations}

In this case there are no topological obstructions to the choice of a singularity-free coset representativel $l(p)$ for all $p \in \Sigma_{t}$, so the method of sections is unnecessary. If $D(\cdot)$ is a UIR of $\mathrm{SU}(2)$ in the space $\mathscr{r}$ (of dimension $(2 s+1)$ corresponding to spin $\mathrm{s}$ ), the induced UIR of $\overline{\mathscr{P}}$ operates on functions $\varphi(\mathrm{p})$ with values in $\mathscr{F}$, i.e. on $(2 \mathrm{~s}+1)$-component wave functions $\varphi_{a}(\mathbf{p})$. The inner product is

$$
\begin{aligned}
\langle\varphi \mid \varphi\rangle & =\int_{\Sigma_{\mathfrak{t}}} \frac{\mathrm{d}^{3} p}{p^{0}} \varphi(\mathbf{p})^{+} \varphi(\mathbf{p}), \\
p^{0} & =(1+\mathbf{p} \cdot \mathbf{p})^{1 / 2}
\end{aligned}
$$

Correspondingly 'the (ideal) basis vectors for $\mathscr{H}$ are $\mid \mathbf{p}$, a) and obey

$$
\left\langle\mathbf{p}^{\prime}, \beta \mid \mathbf{p}, \alpha\right\rangle=p_{0} \delta_{(3)}\left(\mathbf{p}^{\prime}-\mathbf{p}\right) \delta_{\beta \alpha} .
$$

The action of $U(A, a)$ is given by

$$
\begin{aligned}
U(A, a) \cdot \mathbf{p}, \alpha\rangle & =\exp \left(-i a^{\mu} p_{\mu}^{\prime}\right) \sum_{\beta} D\left(l\left(p^{\prime}\right)-{ }^{1} A l(p)\right)_{\beta \alpha}\left|\mathbf{p}^{\prime} . \beta\right\rangle, \\
p^{\prime \mu} & =\Lambda(A)^{\mu}{ }_{\nu} p^{\nu} .
\end{aligned}
$$

The only interesting question is whether $l(p)$ can be chosen so as to satisfy (18):

$$
l(h p)=h l(p) h^{-1}, \quad h \in \mathrm{SU}(2) .
$$


The answer is well known: the choice

$$
l(p)=\left[2\left(1+p^{0}\right)\right]^{-1 / 2}\left(1+\sigma_{\mu} p^{\mu}\right)
$$

does fulfil (54). Therefore if we restrict $A$ in (53)to elements of SU(2), we have the simple action

$$
\begin{aligned}
& U(h)|\mathbf{p}, \alpha\rangle=\sum_{\beta} D(h)_{\beta \alpha}\left|\mathbf{p}^{\prime}, \beta\right\rangle, \\
& \mathbf{p}^{\prime}=R(h) \mathbf{p}, \quad h \in \operatorname{SU}(2) .
\end{aligned}
$$

\subsection{The spacelike representations}

Unlike $\Sigma_{t}$ which is topologically like $\mathbb{R}^{3}, \Sigma_{s}$ has a nontrivial structure. As coordinates for $\Sigma_{s}$ we can use the unit vector $\hat{\mathbf{p}} \in S^{2}$ and $p^{0} \in \mathbb{R}$ : then the 3 -vector $\mathbf{p}$ is

$$
\mathbf{p}=\left(1+\left(p^{0}\right)^{2}\right)^{1 / 2} \hat{\mathbf{p}}
$$

We know in advance that $\Sigma_{s}$ must be expressed as the union $R_{a} \cup R_{b}$ of two open, topologically trivial regions (two will do) to avoid singularities in $l(p)$. The first question is whether $\mathrm{R}$, and $R_{b}$ can be chosen to be invariant under $\mathrm{SU}(1,1)$. For $h \in \mathrm{SU}(1,1)$, the Lorentz transformation $\Lambda(h)$ acts on $p^{0}, p^{1}, p^{2}$, (as an element of $\operatorname{SO}(2,1)$ )and leaves $p^{3}$ invariant. Therefore we make the choices

$$
\begin{aligned}
& R_{a}=\left\{p^{\mu} / p^{\mu} p_{\mu}=1, p^{3}>-1\right\} \\
& R_{b}=\left\{p^{\mu} / p^{\mu} p_{\mu}=1, p^{3}<1\right\}
\end{aligned}
$$

then each of them is invariant under $\operatorname{SU}(1,1)$. The distinguished point $p^{(0)}=(0,0,0,1)$ lies in $R_{a}$. We can now ask for an $l_{a}(p)$ defined free of singularities all over $R_{a}$ obeying

$$
\begin{aligned}
& l_{a}(p) p^{(0)}=p, \\
& l_{a}(h p)=h l_{a}(p) h^{-1}, \quad h \in \mathrm{SU}(1,1)
\end{aligned}
$$

It has been shown elsewhere that such $l_{a}(p)$ can be constructed (Mukunda 1970): an example, strikingly similar to the solution (32)for the $\mathrm{E}(3)$ problem, is

$$
l_{a}(p)=\left[2\left(1+p_{3}\right)\right]^{-1 / 2}\left(1+p^{\mu} \sigma_{\mu} \sigma_{3}\right) .
$$

Next we show by an argument like the one used in $\S 2$ that it is impossible to find an $l_{b}(p)$, smoothly defined all over $R_{b}$, obeying (59). If such an $l_{b}$ existed, then consider the point $-p^{(0)}=(0,0,0,-1) \in R_{b}$ which has the same (homogeneous) stability group $\mathrm{SU}(1,1) \subset \mathrm{SL}(2, \mathrm{C})$ as $p^{(0)}$. Then at the point $-p^{(0)},(59)$ for $l_{b}$ would lead to:

$$
l_{b}((0,0,0,-1))=h l_{b}((0,0,0,-1)) h^{-1}, \quad h \in \mathrm{SU}(1,1),
$$

i.e. $l_{b}((0,0,0,-1))$ must belong to the cornmutant of $\operatorname{SU}(1,1)$ in $\operatorname{SL}(2, C)$. But this cornmutant is trivial, consisting just of the two elements $Z_{2}=\{\mathbb{1},-\mathbb{1}\}$; which then means that $l_{b}((0,0,0,-1))$ cannot carry $p^{(0)}$ to $-p^{(0)}$ :

$$
l_{b}((0,0,0,-1)) \in Z_{2} \Rightarrow l_{b}((0,0,0,-1)) p^{(0)}=p^{(0)} \neq-p^{(0)}
$$


The two desirable conditions (59) can therefore be obeyed on $R_{a}$ but not on $R_{b}$. Under these circumstances, a choice for $l_{b}$ that suggests itself, in analogy to (35) in the $E(3)$ case, is:

$$
l_{b}(p)=l_{a}(-p) \cdot \mathrm{i} \sigma_{2}, \quad p \in R_{b},-p \in R_{a} .
$$

With this $l_{b}(p)$ the transition element $h_{T}(p) \in \mathrm{SU}(1,1)$ can be computed:

$$
\begin{aligned}
h_{T}(p) & =l_{a}(p)-{ }^{1} l_{b}(p) \\
& =l_{a}\left(-p^{0},-p^{1},-p^{2}, p^{3}\right) l_{a}(-p) \cdot i \sigma_{2} \\
& =\left(\begin{array}{ll}
\lambda(p) & \mu(p) \\
\mu(p)^{*} & \lambda(p)^{*}
\end{array}\right) \in \mathrm{SU}(1,1), \\
\lambda(p) & =\left(-p_{1}+i p_{2}\right) /\left(1-p_{3}^{2}\right)^{1 / 2}, \quad \mu(p)=-p^{0} /\left(1-p_{3}^{2}\right)^{1 / 2}, \\
& p \in R_{a} \cap R_{b} .
\end{aligned}
$$

The behaviour of $l_{b}(p)$ under SU $(1,1)$ can also be found; in place of $(59)$, we have:

$$
\begin{aligned}
l_{b}(h) & =l_{a}(-h p) \cdot i \sigma_{2} \\
& =h \cdot l_{a}(-p) h^{-1} \cdot i \sigma_{2} \\
& =h^{\prime} \quad \tau\left(h^{-1}\right), \\
\tau(h)= & \sigma_{2} h \sigma_{2} \in \operatorname{SU}(1,1) .
\end{aligned}
$$

Here, $\tau(\cdot)$ is an outer automorphism on $\operatorname{SU}(1,1)$, the effect on a general element being

$$
\tau\left(\left(\begin{array}{ll}
\lambda & \mu \\
\mu^{*} & \lambda^{*}
\end{array}\right)\right)=\left(\begin{array}{cc}
\lambda^{*} & -\mu^{*} \\
-\mu & \lambda
\end{array}\right) .
$$

Putting together the above results we see that when the spacelikeUIR of $\overline{\mathscr{P}}$, based on the UIR $D(\cdot)$ of $S U(1,1)$ on $\mathscr{V}$, is described in a singularity-free manner using the method of sections, each $|\varphi\rangle$ in $\mathscr{H}$ is represented by a pair of functions $\varphi_{a}(p), \varphi_{b}(p)$ defined respectively on $R, R_{b}$ and taking values in $\mathscr{V}$; they obey the transition rule

$$
\varphi_{b}(p)=D\left(h_{T}(p)\right)^{-1} \varphi_{a}(p), \quad p \in R_{a} \cap R_{b} ;
$$

and with respect to $S U(1,1)$ we can at best achieve

$$
\begin{aligned}
& \left.U(h)|\varphi\rangle=\varphi^{\prime}\right\rangle: \\
& \varphi_{a}^{\prime}(p)=D(h) \varphi_{a}\left(h^{-1} p\right) \\
& \varphi_{b}^{\prime}(p)=D(\tau(h)) \varphi_{b}\left(h^{-1} p\right), \quad h \in \mathrm{SU}(1,1)
\end{aligned}
$$

It is impossible to achieve (19).

The analogues to $(51,52,53,56)$ can be worked out, but we omit the details. 
On $\Sigma_{l}^{(2)}$ however $E(2)$ acts nontrivially. From (71) we see that the choice

$$
a_{\perp}=-p_{\perp} /\left(p^{0}-p^{3}\right)
$$

gives a $\boldsymbol{T}(2)$ transformation that carries $p^{\mu} \in \Sigma_{l}^{(2)}$ into

$$
p^{\prime \mu}=\frac{1}{2}\left(p^{0}-p^{3}, 0,0, p^{3}-p^{0}\right)
$$

Conversely, starting with $(\kappa, 0,0,-\kappa)$ for $\kappa>0$ we can get any desired value for $p_{\perp}$ via a suitable $T(2)$ element:

$$
\begin{aligned}
& (\kappa, 0,0,-\kappa) \in \Sigma_{l}^{(2)} \stackrel{T(2)}{\longrightarrow} \\
& p^{\mu}=\left(\left(p_{\perp}^{2}+4 \kappa^{2}\right) / 4 \kappa, p_{\perp},\left(p_{\perp}^{2}-4 \kappa^{2}\right) / 4 \kappa\right), \quad \kappa>0 .
\end{aligned}
$$

Returning to the three-dimensional picture of $\Sigma_{l}$ : each point on the positive z-axis is $E(2)$ invariant; each point on the negative z-axis expands under $E(2)$ action into a paraboloid of revolution about the z-axis, with vertex on the negative $\mathrm{z}$-axis, and opening out in the direction of increasing z. The open $E(2)$ invariant region $\Sigma_{l}^{(2)}$ is just the union of all these paraboloids:

With this detailed geometricalpicture of the $E(2)$ action on $\Sigma_{l}$ viewed as $\mathbb{R}^{3}-\{0\}$, the claim made earlier can be proved. If $\Sigma_{l}$ is the union of two overlapping open sets $R$, and $R_{b}$, each $E(2)$ invariant, then $\Sigma_{l}^{(1)}$ must be contained in (at least) one of them, say in $R_{b}$. (The possibility that only part of $\Sigma_{l}^{(1)}$ is in $R_{b}$ can be handled in a manner similar to the ensuing argu'ment). Since $R_{b}$ is open, some open region surrounding $\Sigma_{l}^{(1)}$ must also be contained in $R_{b}$. By the $E(2)$ action, since $R_{b}$ is assumed to be $E(2)$ invariant, some open collection of the paraboloids of revolution, forming an open region containing $\Sigma_{l}^{(1)}$, must be in $R_{b}$. But that means that $R_{b}$ is topologically nontrivial, since the origin is excluded: In other words, $R_{b}$ has the same degree of topological nontriviality as $\mathbb{R}^{3}-\{\boldsymbol{0}\}$, if not more. That is, the bundle cannot be trivialized over $\mathbb{R}_{b}$. Any local trivializations of the bundle must use topologically trivial open sets $R_{a}$ and $R_{b}$ of which at least one is not $E(2)$ invariant.

In view of the above result, let us accept the choice of topologically trivial open regions $R_{a}, R_{b}$ according to

$$
\begin{aligned}
& R_{a}=\left\{p^{\mu} / p^{\mu} p_{\mu}=0, p^{0}>0, p^{0}-p^{3}>0\right\}, \\
& R_{b}=\left\{p^{\mu} / p^{\mu} p_{\mu}=0, p^{0}>0, p^{0}+p^{3}>0\right\} .
\end{aligned}
$$

We do have $\Sigma_{l}=R_{a} \cup R_{b}$, and

$$
p \in R_{a} \cap R_{b} \Leftrightarrow p_{\perp} \neq 0
$$

The open set $R_{a}$ is $\Sigma_{l}^{(2)}$, and is $E(2)$ invariant. However $R_{b}$, which contains $\Sigma_{l}^{(1)}$ and in particular the distinguished point $p^{(0)}=(1,0,0,1)$, is not $E(2)$ invariant. Coset representatives $l_{a}(p), l_{b}(p)$ can be chosen smoothly over $R, R_{b}$ respectively. While for $l_{b}(p)$ such a question cannot be posed, for $l_{a}(p)$ we can ask whether it can obey

$$
l_{a}(h p)=h l_{a}(p) h-^{1}, \quad h \in E(2) .
$$

It turns out, however, that this is impossible! $R$, contains four-momenta of the form 


\subsection{The lightlike representations}

The coset space $\Sigma_{l}$, which is just the positive light cone, can be described in three dimensions as

$$
\Sigma_{l}=\left\{\mathbf{p} / p^{0}=|\mathbf{p}|>0\right\}=\mathbb{R}^{3}-(0) .
$$

It is the omission of the origin from $\mathbb{R}^{3}$ that makes the topology of $\Sigma_{l}$ nontrivial. Since $\mathrm{SL}(2, \mathrm{C})$ is a non-trivial $E(2)$ bundle over $\mathrm{C}$, we do need (at least) two open topologically trivial regions $R, R_{b}$ with $\Sigma_{l}=R_{a} \cup R_{b}$, to avoid kinematic singularities in $l(p)$. The first question we ask is whether $R_{a}$ and $R_{b}$ can be chosen to be both $E(2)$ invariant. Surprisingly, the answer is in the negative.

To see this, we analyse in detail the action of $E(2)$ on $\mathrm{C},$. A general element in $E(2)$ is

$$
h=\left(\begin{array}{ll}
\lambda & \mu \\
0 & \lambda^{*}
\end{array}\right), \quad|\lambda|=1, \quad \mu \in \mathbb{C} .
$$

The phase $\lambda$ produces spatial rotations about,the z-axis, which act very simply on $\Sigma_{l}$. The complex parameter $\mu$ is just the translation part, $T(2)$, in $E(2)$. If we write $\mu=a_{1}-i a_{2}$ and treat $\mathrm{a},=(a, a$,$) as a transverse 2-vector, we find the following action$ on a general $p^{\mu} \in \Sigma_{l}$ :

$$
\begin{aligned}
& h=\left(\begin{array}{cc}
1 & a_{1}-i a_{2} \\
0 & 1
\end{array}\right) \in E(2), \quad p^{\prime}=\Lambda(h) p \\
& p^{0 \prime}-p^{3 \prime}=p^{0}-p^{3} \\
& p^{0 \prime}+p^{3 \prime}=p^{0}+p^{3}+2 a_{\perp} \cdot p_{\perp}+\left(p^{0}-p^{3}\right) a_{\perp}^{2} \\
& p_{\perp}^{\prime}=p_{\perp}+\left(p^{0}-p^{3}\right) a_{\perp} .
\end{aligned}
$$

In the three-dimensional picture of $\Sigma_{t}$ given by (69) we see: each point on the positive zaxis is $\boldsymbol{E}(2)$ invariant; each point not on the positive z-axis is either on the negative $z$ axis or can be transformed to such a point uniquely by a suitable choice of $T(2)$ parameters $a_{\perp}$. This motivates the definition of two disjoint subsets of $\Sigma_{l}$, together making up $\Sigma_{l}$, in this way:

$$
\begin{aligned}
& \Sigma_{l}=\Sigma_{l}^{(1)} \cup \Sigma_{l}^{(2)} \\
& \Sigma_{l}^{(1)} \cap \Sigma_{l}^{(2)}=\phi \\
& \Sigma_{l}^{(1)}=\{(0,0, \kappa) / \kappa>0\} \\
& \Sigma_{l}^{(2)}=\left\{\left(p_{\perp}, p_{3}\right) / \text { either }\left|p_{\perp}\right|>0 \text { or } p_{\perp}=0, p_{3}<0\right\} .
\end{aligned}
$$

In the Minkowski sense, $\Sigma_{l}^{(1)}$ and $\Sigma_{l}^{(2)}$ correspond respectively to $p^{0}-p^{3}=0$ and $p^{0}-p^{3}>0$; so each of them is $E(2)$ invariant, However, while $\Sigma_{l}^{(2)}$ is an open subset of $\mathrm{C}, \Sigma_{l}^{(1)}$ is not, and this is the source of the problem. Four-vectors $p^{\mu} \in \Sigma_{l}^{(1)}$ are individually $E(2)$ invariant:

$$
p^{\mu} \in \Sigma_{l}^{(1)} \Leftrightarrow p^{\mu}=\kappa p^{(0) \mu}=(\kappa, 0,0, \kappa), \quad \kappa>0 .
$$


but with the definitions of $R, R_{b}$ and $h_{T}(p)$ appropriate for the lightlike situation. Under the action of an element of $E(2)$, we have:

$$
\begin{aligned}
& U(h)|\varphi\rangle=\left|\varphi^{\prime}\right\rangle: \\
& \varphi_{a}^{\prime}(p)=D\left(\tau^{\prime}(h)\right) \varphi_{a}\left(h^{-1} p\right), \quad h \in E(2) .
\end{aligned}
$$

For $\varphi_{b}^{\prime}$ we have no simple formula since $E(2)$ does not leave $R_{b}$ invariant; in fact $\varphi_{b}^{\prime}$ involves both $\varphi_{a}$ and $\varphi_{b}$, reminding one of the indecomposability of the lightlike representations of $\overline{\mathscr{P}}$ found in another context (Matthews et al 1974).

\section{Concluding remarks}

Motivated by the quantum mechanical description of states in the field of a magnetic monopole, we have analysed the UIR's of the (covering groups of the) Euclidean group $E$ (3) and Poincare group $\mathscr{P}$, to see how kinematic singularities can be avoided in the wave functions occurring in these UIR's. We have answered the following questions which come up naturally when these UIR's are constructed by the inducing procedure:

(a) In those cases where the group $G(E(3)$ or $\mathscr{P})$ is a nontrivial principal fibre bundle on the relevant coset space $\Sigma=G / H$, what are natural choices of open topologically trivial regions of $\Sigma$ over each of which the bundle can be trivialized?

(b) Can each of these regions be chosen to be individually invariant under $\mathrm{H}$, the homogeneous part of $H$ ?

(c) In the UIR of $\mathrm{G}$ that results by inducing, starting from a UIR $D(h)$ of $\boldsymbol{H}$, , can the action of elements of $H_{0}$ be made as simple as possible and to involve $D(h)$ itself? Can the choices of coset representatives be adjusted to achieve this?

Leaving aside the case of $E(3)$, the UIR s of $\overrightarrow{\mathscr{P}}$ have been found to behave as follows: In the timelike representations, as is well known, the bundle is trivial, and globally smooth choices of coset representatives $l(p)$ do exist. Moreover $l(\not)$ can be chosen so that for elements $h \in H_{0}=\mathbf{S U}(2)$ the Wigner rotation is not momentum dependent. Then the UIR $D(h)$ of $\mathbf{S U}(2)$ used in the inducing construction directly describes the $\mathrm{SU}(2)$ behaviour of wave functions. For spacelike representations, two regions $R_{a}$ and $R_{b}$ in $\Sigma$ are needed, as in the monopole and $E(3)$ problems, and we can choose them to be individually $S U(1,1)$ invariant. Over $R$, the coset representative $l_{a}(p)$ can be chosen to have the simplifying property (18); the one over $R_{b}$ cannot. The lightlike representations show further complexity. Two regions $\mathrm{R}, R_{b}$ are needed to trivialize the bundle, but of these only $R_{a}$ is $E(2)$ invariant. Moreover, even on $R_{a}$ we cannot pick a smooth coset representative $l_{a}(p)$ obeying (18). Thus from the view point of this paper we see a gradual increase in the intricacy of the topological structure and group theoretical behaviour as we go from $t$ to $s$ to 1 .

We have not given general formulae, in the $s$ and $l$ cases, for the effect of a general $U(g)$ on $\varphi_{a}$ and $\varphi_{b}$. One expects a mixing of these sections, easy in principle, but tedious in practice to work out. For a comparison, the case of monopole harmonics has been worked out in detail by $\mathrm{Wu}$ and Yang.

Finally we would like to collect together and draw attention to the specific coset representatives we have found in the case of the Poincare group, for the $s$ and 1UIR's. 
Including also the well-known choice in the timelike case, the complete list is:

$$
\begin{array}{ll}
t: & l(p)=\left[2\left(1+p^{0}\right)\right]^{-1 / 2}(1+p \cdot \sigma) ; \\
s: & l_{a}(p)=\left[2\left(1+p_{3}\right)\right]^{-1 / 2}\left(1+p \cdot \sigma \sigma_{3}\right), \quad p_{3}>-1, \\
& l_{b}(p)=\left[2\left(1-p_{3}\right)\right]^{-1 / 2}(1-p \cdot 663) i \sigma_{2}, \quad p_{3}<1 ; \\
l: & l_{a}(p)=\left[2\left(p^{0}-p^{3}\right)\right]^{-1 / 2}\left(1+\sigma_{3}-p \cdot \sigma\right) \sigma_{2}, \quad p^{0}-p^{3}>0, \\
& l_{b}(p)=\left[2\left(p^{0}+p^{3}\right)\right]^{-1 / 2}\left(1-\sigma_{3}+p \cdot \sigma\right), \quad p^{0}+p^{3}>0 .
\end{array}
$$

It would be interesting to see if there is some general argument leading to such similar forms in these widely differing situations, which then might be meaningful in higher dimensions.

\section{References}

Balachandran A P, Marmo G, Skagerstam B S and Stern A 1980 Nucl. Phys. B162 385

Biedenharn L C and Louck J D 1981 The Racah-Wigner Algebra in Quantum Theory Encyclopaedia of Mathematics and its Applications (Reading, Mass.: Addison-Wesley) Vol. 9, Chap. 5, Topic 2

Coleman S 1981 The magnetic monopole fifty years later, Lectures delivered at the International School of Subnuclear Physics, Ettore Majorana, Harvard University Preprint HUTP-82/A032

Dirac P A M 1931 Proc. R. Soc. London A133 60.

Goddard P and Olive D I 1978 Rep. Prog. Phys. 411357

Isham C J 1983 Relativity, Groups and Topology II, Les Houches, Session XL, Course 11, Section 5.8 (Eds)

B S Dewitt and R Stora (Amsterdam: North-Holland) p. 1266

Mackey G W 1968Induced representations of groups and quantum mechanics (N. Yark and Amsterdam:W. A. Benjamin Inc., Torino: Editore Boringhieri)

Matthews P M, Seetharaman M and Simon M T 1974 Phys. Rev. D9 1706

Mukunda N 1970 Ann. Phys. (N.Y) 61329

Pauli W 1965 Ergebnisse der Exacten Naturwissenschaften Band 37 p. 85 (Berlin, Heidelberg, New York: Springer-Verlag)

Wightman A S 1960Relations de dispersion et particules elementaires, Les Houches (Paris: Hermann) p. 159

Wigner E 1939 Ann. Math. 40149

Wu T T and Yang C N 1975 Phys. Rev. D12 3845

Wu T T and Yang C N 1976 Nucl. Phys. B107 365 